\title{
The Minimum Quality Criteria Set (QI-MQCS) for critical appraisal: advancing the science of quality improvement
}

\author{
Lisa V Rubenstein ${ }^{1,2,3^{*}}$, Susanne Hempel ${ }^{2}$, Jodi L Liu², Margie J Danz ${ }^{1,2}$, Robbie Foy ${ }^{4}$, Yee-Wei Lim ${ }^{5}$, Aneesa Motala ${ }^{2}$ \\ , Paul G Shekelle $e^{1,2}$
}

From 7th Annual Conference on the Science of Dissemination and Implementation in Health

North Bethesda, MD, USA. 8-9 December 2014

\section{Objective}

Effective learning across related scientific investigations through evidence synthesis is critical to promoting evidence-based approaches to healthcare. Synthesis of findings from quality improvement intervention (QII) publications, however, poses challenges. We aimed to develop a critical appraisal instrument (the Minimum Quality Criteria Set or QI-MQCS) to promote identification, dissemination and implementation of findings from high quality QII evaluations.

\section{Methods}

We convened a 9 person expert panel to guide QII evidence synthesis methods development through a one year iterative telephone, survey and in-person panel process. We developed and empirically tested electronic search and screening methods for identifying QII publications, and a critical appraisal instrument. Finally, we iteratively tested and improved QI-MQCS psychometric properties based on review of 54 electronically searched and systematically screened QII articles.

\section{Results}

Panelists agreed QI-MQCS should focus on QII specific domains, not evaluation design criteria. The 16 QI-MQCS domains address Organizational Motivation, Intervention Rationale, Intervention Description, Organizational Characteristics, Implementation, Study Design, Comparator Description, Data Sources, Timing, Adherence / Fidelity, Health Outcomes, Organizational Readiness, Penetration /
Reach, Sustainability, Spread, and Limitations. The median inter-rater agreement for QI-MQCS items was kappa 0.57 (83\% agreement). Items discriminated between studies in terms of quality (median criteria met $67 \%$ ). Internal consistency measures indicated coherence without excessive conceptual overlap (Cronbach's alpha $=0.60$, absolute mean inter-item correlation $=0.19$ ). The critical appraisal instrument is accompanied by a user manual detailing What to consider, Where to look, and How to rate.

\section{Conclusions}

The QI-MQCS had acceptable psychometric properties for critical appraisal, and can support systematic review of diverse QII evaluations. It is a ready-to-use critical appraisal tool accompanied by a user manual and empirically tested forms and methods.

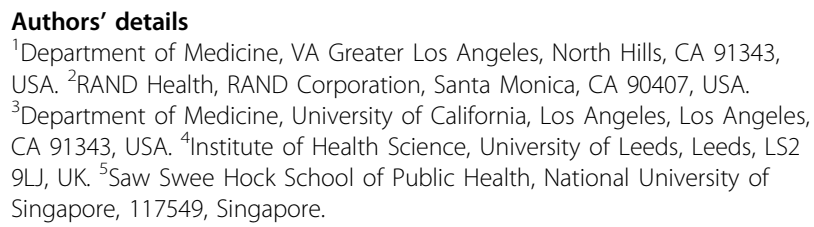

Published: 20 August 2015

doi:10.1186/1748-5908-10-S1-A19

Cite this article as: Rubenstein et al:: The Minimum Quality Criteria Set (QI-MQCS) for critical appraisal: advancing the science of quality improvement. Implementation Science 2015 10(Suppl 1):A19.

\footnotetext{
* Correspondence: Lisar@rand.org

'Department of Medicine, VA Greater Los Angeles, North Hills, CA 91343, USA

Full list of author information is available at the end of the article
} 